\title{
COMMISSION 5: DOCUMENTATION (DOCUMENTATION)
}

\author{
Report of Meeting, 24 August 1973
}

President: J. B. Sykes.

SECRETARY: F. Henn.

1. The President's report was approved on the motion of Fricke.

2. Reports on Astronomy. After discussing a letter from the General Secretary, the Commission carried unanimously the following resolution:

Commission 5 reiterates its resolution passed at the XIV General Assembly (Transactions XIVB, p. 86) concerning the desirability of continuing to publish the Reports on Astronomy in one volume. Commission 5 wishes in particular for its own report to continue to be published by the IAU.

It was regarded as understood that there would continue to be limitations on the length of reports, and means of obtaining the report of each Commission separately, and that the reports should be critical rather than merely enumerative.

3. Composition of the Commission. The President reported the deaths of four members (Alter, Heinemann, Luplau Janssen, and Zinner) and the resignation of Belorizky, Chandrasekhar, Sticker, and Velghe. P. Lantos (Meudon) was proposed as a new member subject to membership of the IAU, and Mrs C. A. Bain (Dudley Observatory) as a new consulting member.

4. Officers of the Commission, 1973-76. Ogorodnikov having declined the succession to the Presidency, Pecker was elected President and Heintz Vice-President, with an Organizing Committee of five other members: Henn, Kleczek, Shcherbina-Samojlova, Sykes and Wilkins. The name of Maxwell was later added to the Organizing Committee (see report of second joint meeting, below).

5. Working Groups. The question of a Working Group on Nomenclature, to coordinate nomenclature e.g. for galaxies, double stars, and formations on the Moon, was discussed. It was agreed to ascertain the conclusions of other Commissions discussing nomenclature problems.

The Working Group on Numerical Data, established by the Union in 1970, was in need of attachment to a Commission. It was agreed that this should become a Working Group of Commission 5 (Chairman: G. A. Wilkins), with the name 'Working Group on the Documentation, Exchange and Retrieval of Numerical Data'.

The Working Group on Universal Decimal Classification 52 (Astronomy) was continued.

Another Working Group was later added (see report of second joint meeting, below).

6. Working Rules. The President introduced some draft working rules of Commission 5, prepared in accordance with the wish of the Executive Committee. It was agreed that these should be discussed by the Organizing Committee.

7. ICSU Abstracting Board. The President reported his recent work in the Board. The text of a leaflet on secondary (abstracting and indexing) services prepared by Wilkins for wide distribution to astronomers and others was presented and generally approved. Similar leaflets already exist for services in physics and biology.

8. Classification. Some progress was reported concerning a joint committee with FID on the revision of UDC 52, but none towards the establishing of a common classification for use by secondary services. It was agreed that work on unification of classification schemes and development of multilingual thesauri deserves priority.

9. Astronomical Dictionary. The President reported that the Astronomical Dictionary by Kleczek (1961) had been revised and greatly enlarged to 15000 terms ( 91 sections) and eight languages. The Commission carried unanimously the following resolution:

Commission 5 has learnt with pleasure of the approaching completion in typescript of a revised edition of the Astronomical Dictionary by J. Kleczek, containing a greatly increased number of 
terms over the whole field of astronomy and space science and extended to include eight languages. Commission 5 urges institutions in Czechoslovakia and elsewhere to assist in the publication and distribution of this valuable work.

10. Ancient books. The President reported the completion of the catalogue of astronomical books of the fifteenth and sixteenth centuries in European observatory libraries.

11. List of non-commercial publications. The President reported the forthcoming third edition of the Utrecht bibliography.

12. IAU Symposium and Colloquium proceedings. It was noted that the Union publishes a list indicating the availability of these, supplemented by notes in the Information Bulletin.

\section{Joint Meeting of Commissions 5, 9, 38 and 46}

The meeting took place on 27 August under the chairmanship of J. B. Sykes. Its purpose was to continue consideration of the construction and publication of a world list of observatories, astronomers, instruments and programmes.

The chairman reported his failure to get adequate support from National Committees for Astronomy for the scheme discussed in 1970 (Transactions XIVB, p. 89).

After discussion of the forms in which information was already available, it was agreed that Heintz should seek to collate materials for the United States and to establish regional correspondents for similar work regarding other countries.

\section{Joint Meeting of Commissions $5,36,38$ and 46}

The meeting took place on 29 August under the chairmanship of J. B. Sykes.

After discussion of the need to improve uniformity of editorial policy in astronomical journals, and reference to the existence of associations of editors in various other disciplines and geographical areas, it was resolved to form a Working Group of Commission 5 (Chairman: A. Maxwell), with the name of 'Working Group on Editorial Policy' and up to ten members. The terms of reference of the Working Group are:

(1) To investigate the possibilities of collaboration between editors of astronomical journals in such matters as style recommendations, relationships with secondary (abstracting and indexing) journals, publishers, authors, etc.

(2) To advise on the revision of the IAU Style Book.

After discussion of the economics of publication, the competition between quality and cheapness of production, and available information about methods of inexpensive publication, it was resolved to recommend to the Executive Committee the initiation or continuation of investigations with non-profit organizations such as Annual Reviews Inc., considering in particular: (1) the feasibility of establishing an independent non-profit IAU publishing house, (2) savings obtainable by the use of soft covers, cheaper paper, etc., (3) the cheap separate availability of reprints of proceedings of Commissions, Joint Meetings, etc. 